\title{
Meeting the needs of orphans and other vulnerable children: Learnings from Project SOAR
}

Project SOAR

Follow this and additional works at: https://knowledgecommons.popcouncil.org/departments_sbsr-hiv

Part of the Demography, Population, and Ecology Commons, Family, Life Course, and Society Commons, International Public Health Commons, and the Maternal and Child Health Commons How does access to this work benefit you? Let us know!

\section{Recommended Citation}

Project SOAR. 2019. "Meeting the needs of orphans and other vulnerable children: Learnings from Project SOAR," Project SOAR Summary Brief. Washington, DC: Population Council. 


\section{Meeting the Needs of Orphans and \\ Other Vulnerable Children}

\section{Learnings from Project SOAR}

Children are disproportionately affected by the HIV epidemic, particularly those who have lost one or both parents to HIV, are being raised by a sick caregiver, or are living with HIV. These orphans and other vulnerable children (OVC) often have a range of unmet needs, such as education, food security, psycho-social support, and access to health care. They also are particularly vulnerable to abuse and exploitation, as well as more likely to engage in unsafe or risky behaviors that place them at elevated risk of HIV. Project SOAR is documenting the unique vulnerabilities of these children and their caregivers, and examining strategies to mitigate the impacts of HIV on them.

\section{OUR RESEARCH ACTIVITIES}

\section{Strengthening community-based services for children and families affected by HIV: an evaluation of the Zambia Family Program}

The Zambia Family (ZAMFAM) program aims to improve the care and resilience of OVC and their households through child- and family-focused services. Project SOAR conducted a survey among beneficiaries in four provinces before ZAMFAM began scaling up activities. The findings provide a deeper understanding of the needs of families of OVC and the gaps in service provision, as well as recommendations for strengthening care and support strategies for OVC in Zambia.

\section{What did we find?}

- Substantial proportions of OVC had not been linked to health, administrative, or educational services. As a result, OVC had low vaccination rates, high rates of sickness, and limited access to birth certificates.

- The HIV status of only about half of the OVC was known to their caregiver.

- Economic and food insecurity were major concerns for OVC and their families.

- OVC and their caregivers, who are often older women with little or no formal education, experience limited social support.

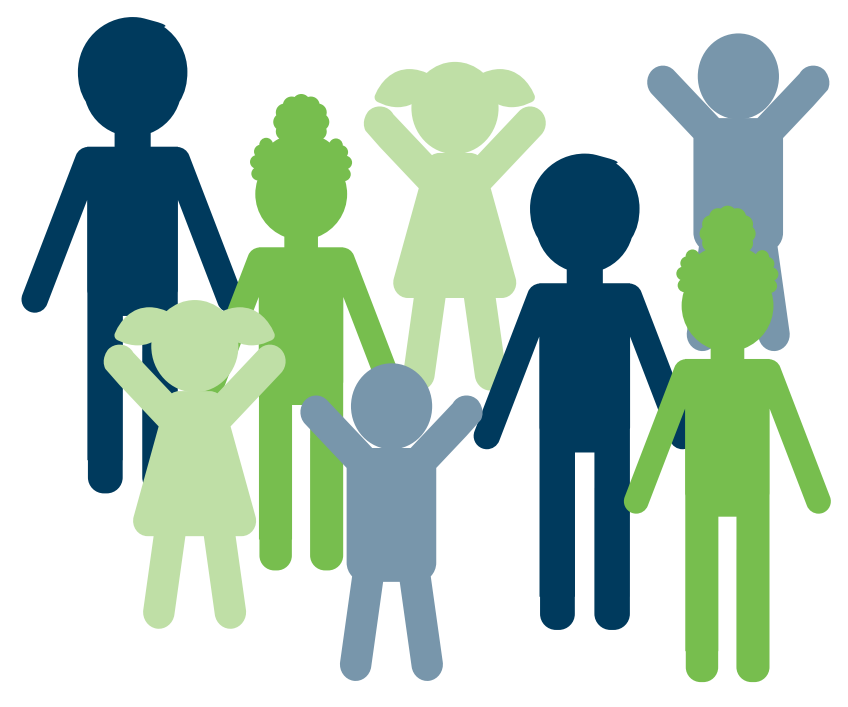

\section{What did we recommend?}

- Strengthen OVC households' linkages to health facilities and/or implement outreach efforts to address the health needs of OVC.

- Address resource constraints to meet educational expenses and reduce late enrollment and early dropout.

- Incorporate sustainable approaches to ensure access to food through economic empowerment, as well as improved agricultural production and increased animal husbandry in rural areas.

- Provide guidance to OVC caregivers to help them access appropriate services, including social support, for themselves and for the children in their care.

Project SOAR also undertook a prospective cohort study of young people living with HIV (YPLHIV) and their primary caregivers/guardians, collecting two rounds of data over a one-year period to determine the effects of ZAMFAM on the well-being of YPLHIV and their households.

\section{What did we find?}

- The vast majority of YPLHIV in both intervention and comparison areas adhered to treatment and stayed in care at both rounds of data collection. 
- Self-reported viral load testing increased among YPLHIV in both areas between survey rounds, although it still remained low.

- Access to psychosocial support increased among older YPLHIV in both areas; however, many younger and older children continued to be left out.

- Caregivers in ZAMFAM areas reported significant improvements in financial capacity, joint decisionmaking, and having help when sick.

- Food insecurity, sickness, and school attendance continued to be major problems among YPLHIV and their households, with little change between rounds.

\section{What did we recommend?}

- Strengthen outreach by community health workers and counselors to further improve psychosocial and health support to YPLHIV and their households.

- Sensitize primary caregivers and community health workers on the importance of viral load testing for YPLHIV to ascertain whether they are virally suppressed.

- Enhance the capacities of caregivers to seek timely care for sick YPLHIV at health facilities.

- Strengthen community strategies to improve financial and food security in the most vulnerable households.

\section{What has been the impact of the SOAR ZAMFAM studies?}

- The research team established a technical advisory group that was chaired by the Permanent Secretary in the Ministry of Community Development and Social Welfare, with participation from ZAMFAM implementing partners and other relevant stakeholders. This set the stage for the study findings to be recognized as a key source of evidence on the situation of OVC/YPLHIV in the country.

- ZAMFAM implementing partners have used the results from the two studies to improve programming. Specifically, they have:

- Fostered an increase in birth certificates to OVC.

- Promoted the importance of HIV status awareness among households, resulting in a reduction of OVC with unknown HIV status by almost 50 percent.

- Focused on improving living standards of OVC and supporting caregivers.

\section{Research partners}

Population Council and ZAMFAM

\section{Resources}

Strengthening community-based services for children and families affected by HIV: an evaluation of the Zambia Family Program (Activity Brief) projsoar.org/resources/zambia-zamfam-actbrief/

Benchmark assessment of orphaned and vulnerable children in areas of the Zambia Family (ZAMFAM) Project (Final Report) projsoar.org/resources/zamfam-benchmark-report/

Health and socioeconomic well-being of young people living with HIV in Zambia: evaluating the ZAMFAM Project (Results Brief)

projsoar.org/resources/health-and-socioeconomicwell-being-of-young-people-living-with-hiv-in-zambiaevaluating-the-zamfam-project/

Health and socioeconomic well-being of young people living with HIV one year after implementation of the ZAMFAM Project (Final Results Brief) projsoar.org/resources/zambia-zamfam-youngplhivfirstyr-resbrief/

\section{Project YES! (Youth engaging for success): a peer mentoring program to transition youth to HIV self-management in Zambia}

As adolescents and young adults living with HIV age into adulthood, they must navigate the transition to HIV self-management and adult care. This is a complex process, particularly for young people who must manage a chronic illness such as HIV. As a result, many YPLHIV experience challenges adhering to antiretroviral treatment (ART), leading to poor viral suppression and a greater risk of HIV-related mortality. Project SOAR is refining and testing a peer-mentoring strategy to support youth as they transition to, and engage in, selfmanagement and adult HIV care and treatment.

\section{What did we find?}

At the beginning of the study:

- Nearly three-quarters of male and female YPLHIV reported being a victim of violence (psychological, physical, or sexual) in the past year.

- Experiences of violence. negatively impacted on their mental health, medication regimen, and clinic appointments

- Feelings of guilt, shame, or worthlessness because of their HIV diagnosis was common among YPLHIV.

2 | Meeting the needs of orphans and other vulnerable children 
- More than one-third of participants were not virally suppressed.

- Most YPLHIV not virally suppressed had evidence of drug resistance.

\section{What's next?}

Project YES! is using these findings to address violence, self-stigma, and viral suppression as part of its peer mentoring strategy. Final results on the effectiveness of the strategy will be available in late 2019 .

\section{Research partners}

Johns Hopkins Bloomberg School of Public Health and Arthur Davison Children's Hospital, Ndola, Zambia

\section{Resources}

Project YES! Youth Engaging for Success: A peer mentoring program to transition youth to HIV selfmanagement in Zambia (Activity Brief) projsoar.org/resources/zambia-yes-actbrief/

Youth living with HIV in Zambia: Interpersonal violence, self-stigma, and viral suppression (Project SOAR Results Brief) projsoar.org/resources/zambia-yes-violence-stigmaresbrief/

\section{Evaluation of the "One Community" program in Malawi}

The One Community (One-C) program is a communitybased initiative that seeks to increase utilization of HIV prevention, care, and treatment services among high-risk populations, improve linkages to services at the community level, and encourage the adoption of HIV-risk reduction behaviors. Project SOAR and the University of Malawi, College of Medicine, are collecting three rounds of data over a two-year period to monitor key HIV prevention, care, and treatment outcomes among populations targeted by the One-C program, including OVC and their caregivers, in southern Malawi. In addition, the study investigated depressive symptoms among OVC caregivers in the first survey round.

\section{What did we find?}

Among caregivers of OVC:

- Most reported experiencing at least three (out of five) depressive symptoms sometimes or often in the four weeks prior to the interview.
- Being HIV positive or never having tested for HIV were associated with a greater reporting of depressive symptoms.

- Social support was protective for depressive symptoms.

- Depressive symptoms among caregivers living with HIV were associated with reported lower ART adherence.

\section{What did we recommend?}

- Strengthen social networks in programming for caregivers of vulnerable children.

- Address mental health issues in adherence counseling for HIV-positive OVC caregivers.

\section{What's next?}

An analysis of changes in key indicators over the three rounds of data collection.

\section{Research partners}

Population Council; The University of Malawi, College of Medicine; and the One-C Program

\section{Resources}

Evaluation of the "One Community" program in Malawi (Activity Brief) projsoar.org/resources/malawi-one-cmty-actbrief/

Project SOAR. 2018. "How common are depressive symptoms among caregivers of vulnerable children in Malawi, and what factors are associated with their occurrence? (Results Brief)

projsoar.org/resources/malawi-onec-depression-ovccaregivers-resbrief/

\section{Mixed methods study of support clubs for Haitian children and adolescents living with HIV}

Helping children and young adults living with HIV adhere to ART regimens and maintain viral suppression remains a critical challenge. Community support groups, in the form of adherence clubs, have emerged as a promising strategy and have been documented in a variety of settings for adults and children. The BEST project Kids Club program in Haiti provides a promising platform for further study of adherence and support groups among children and young adults. Given that the clubs have been operating for more than five years, much can be learned from the project's experiences in 
implementing them. Project SOAR is systematically and critically examining the program's operations, costs, and effects on club participants and their families. Findings will help inform future programming in Haiti and globally.

\section{What's next?}

Study results will be available late 2019 .

\section{Research partners}

Palladium, Société d'Études et de Formation en Information Stratégique, and Caris Foundation

\section{Resources}

Mixed methods study of implementation, outcomes, and costs of support clubs for Haitian children and adolescents living with HIV (Project SOAR Activity Brief) projsoar.org/resources/haiti-cag-ovc/

\section{Identifying promising interventions to support engagement and adherence to treatment among OVC in sub-Saharan Africa}

Various strategies-including community support groups-are currently implemented in countries around the world to reduce the barriers to engagement in care and adherence to ART and to support YPLHIV to achieve viral suppression. SOAR is conducting a systematic review of such interventions among OVC to help inform national policy and donor-funded programs in sub-Saharan African countries. The results will address the following research questions:

- What were the underlying theories and assumptions about why these programs work, and what appear to be the mechanisms and associated contextual influences that led to their intended outcomes?

- What were the interventions for OVC in various countries and what were their outcomes?

- Which critical contextual factors influenced intervention implementation outcomes?

\section{What's next?}

The review will be completed mid-2019.

\section{Research partners}

University of North Carolina, Chapel Hill

\section{Evaluating an HIV risk screening tool among OVC in Tanzania}

Identifying children and adolescents for HIV testing has largely been conducted at health facilities, where inpatient status or clinical symptom screening are often used as risk screening criteria. This approach does not systematically reach OVC served through communitybased programs, if they do not regularly access facility services. Equipping community cadres with an appropriate tool to screen OVC at the community level could address this gap. Project SOAR is conducting a cross-sectional study to validate an HIV risk screening tool for OVC and to assess its feasibility and acceptability within community and facility settings. Integrating HIV risk screening into OVC programs could contribute to national and international "90-90-90" goals through improved identification of HIV-positive children/adolescents (i.e., testing yield) and linkage to treatment.

\section{What's next?}

Study results will be available late 2019 .

\section{Research partners}

Elizabeth Glaser Pediatric AIDS Foundation; National AIDS Control Program and Social Welfare/Community Unit; Ministry of Health, Community Development, Gender, Elderly and Children; Pact Tanzania

\section{Resources}

Evaluating an HIV risk screening tool among orphans and other vulnerable children in Tanzania (Activity Brief)

projsoar.org/resources/tanzania-ovc-screening-toolactbrief/

Suggested citation: Project SOAR. 2019. "Meeting the needs of orphans and other vulnerable children: Learnings from Project SOAR," Project SOAR Summary Brief. Washington, DC: Population Council.
Project SOAR is a five-year (September 2014-September 2019) cooperative agreement funded by the U. S. President's Emergency Plan for AIDS Relief and the U. S. Agency for International Development (Agreement No. AIDOAA-A-14-00060). The contents of this brief are the sole responsibility of Project SOAR and Population Council and do not necessarily reflect the views of PEPFAR, USAID, or the United States Government.

Population Council leads the Project SOAR consortium in collaboration with Avenir Health, Elizabeth Glaser Pediatric AIDS Foundation, the Johns Hopkins University, Palladium, and The University of North Carolina at Chapel Hill.
Project SOAR/Population Council

4301 Connecticut Avenue, NW, Suite 280

Washington, DC 20008

Tel: +12022379400

e-mail: ProjectSOAR@popcouncil.org projsoar.org

()Population Council, May 2019 